\title{
Apicogénesis en canino permanente joven con resorción intracoronal pre eruptiva: reporte de caso
}

\author{
Apexogenesis in young permanent canine with pre-eruptive intracoronal resortion: case report
}

\author{
Adriana Patricia Bravo Zhunio ${ }^{1, a, 2, b}$, David Alexis Díaz Sánchez ${ }^{3, a, 4, b}$, Kasandra Verónica Yupanqui Barrios 3 3,a,5,c, \\ Carlos Enrique Mendiola Aquino ${ }^{1, \mathrm{~d}, 6, \mathrm{e}, \mathrm{f}, \mathrm{7c}}$
}

\section{RESUMEN}

Los dientes permanentes presentan desarrollo y formación radicular incompleta al momento de su erupción, el tejido pulpar es el encargado de culminar el proceso de formación radicular por intermedio de los odontoblastos que producen dentina, si la misma se ve afectada por traumatismo, lesión cariosa, resorción intracoronal pre eruptiva (PEIR por sus siglas en inglés) y/o fractura, se altera la vitalidad e incluso puede llegar a producirse necrosis pulpar, lo que ocasionará una interrupción de la formación radicular normal. Para el tratamiento de dientes permanentes jóvenes e inmaduros que han sufrido algún tipo de lesión, pero que aún mantienen la vitalidad pulpar, el procedimiento de apicogénesis con agregado de trióxido mineral (MTA) puede ser considerado como una opción viable, debido a la biocompatibilidad y capacidad de inducir la formación de tejidos duros. En el presente reporte de caso se presenta una pieza dental con antecedente de PEIR, tratada mediante apicogénesis con MTA, lográndose resultados favorables en cuanto al engrosamiento de paredes dentinales, desarrollo radicular y mantenimiento de la vitalidad pulpar.

\section{PALABRAS CLAVE: Apicogénesis, ápice radicular inmaduro, cierre apical, pulpotomía, hidróxido de calcio, MTA}

\section{SUMMARY}

The permanent teeth have an incomplete development and root formation at the time of its eruption, the pulp tissue is responsible for completing the process of root formation by means of odontoblasts that produce dentin, if it is affected by trauma, carious lesion, Pre-eruptive intracoronal resorption (PEIR) and / or fracture, pulp vitality is altered and pulp necrosis may even occur, which will cause an interruption of the normal radicular formation. For

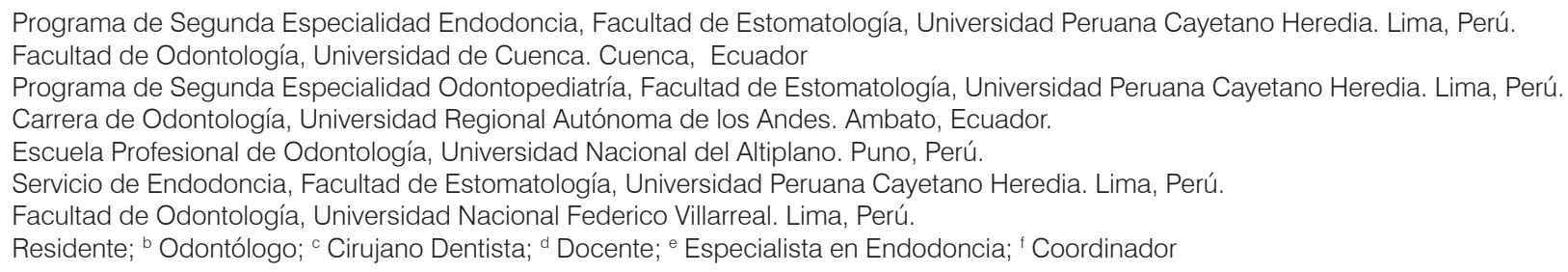


the treatment of immature permanent teeth that have suffered some type of injury, but still maintain pulp vitality, the procedure of apexogenesis with added mineral trioxide (MTA) can be considered as a viable option, due to the biocompatibility and capacity of induce the formation of hard tissues. In this case report we present a tooth with a history of PEIR, treated by apexogenesis with MTA, achieving favorable results in terms of thickening of dentinal walls, root development and maintenance of pulpal vitality.

\section{KEY WORDS: Apexogenesis, immature root apex, apical closure, pulpotomy, calcium hydroxide, MTA}

\section{INTRODUCCIÓN}

La resorción intracoronal pre-eruptiva (PEIR) antes conocida como caries eruptiva, oculta o intrafolicular (1), se presenta como una resorción asintomática idiopática en un diente no erupcionado (2), aparece dejando intacta la capa externa del esmalte de manera espontánea, diferenciándose de una lesión cariosa en que la imagen radiolúcida inicia en la superficie externa del esmalte. No hay invasión microbiana en la lesión pre eruptiva, debido a que la pieza dentaria se encuentra encapsulada en su saco pericoronario, por ende, ha dejado de considerarse como una variante de caries (2). Se ha informado que PEIR es un hallazgo aislado ya que es inaccesible el examen visual $(3,4)$, de aparición más frecuente en primeros molares mandibulares y maxilares, segundos premolares y segundos molares mandibulares permanentes(2,3,5-11).

Se ha informado que la prevalencia de PEIR varía entre $1,55 \%$ y $6 \%$. (2,12-17). No se ha encontrado asociación con el sexo, la raza, el estado médico, la enfermedad sistémica o la administración de suplementos de flúor $(12-14,18)$. Por lo general, un solo diente se ve afectado (19), casi la mitad de estas lesiones se extienden a no más de dos tercios del grosor de la dentina $(1,12,14,20)$.

En la patogénesis se menciona que histológicamente la resorción comienza intracoronalmente en la dentina del diente no erupcionado, causados por invaginación de odontoclastos a través de defectos en el esmalte. Se ha sugerido que la resorción puede ocurrir si hay fracturas en el epitelio reducido del esmalte que envuelve la corona del diente en desarrollo; estas microperforaciones pueden conducir al contacto entre el tejido conectivo y el esmalte dentario. El tejido conectivo, con sus canales de vascularización, puede alterar el desarrollo normal de la corona. PEIR aparece como áreas de degeneración de los túbulos dentinarios que son reemplazados por tejido conectivo vascular $(11,12)$.
McNamara y Seow mencionan que, al emerger el diente afectado a la cavidad oral, las condiciones para el desarrollo rápido de lesiones cariosas se vuelven adecuadas $(18,21)$.

Una afectación pulpar evidentemente es mucho más probable y cuando esta sucede, el tratamiento de dientes inmaduros con pulpa vital tradicionalmente se realiza a través de la apicogénesis con hidróxido de calcio (22), este procedimiento ha estado vigente por décadas, el cual permite el crecimiento radicular continuo y el cierre apical en un periodo de tiempo largo. En la actualidad existen otros materiales biocompatibles como el agregado trióxido mineral (MTA) y métodos que permiten la estimulación de los odontoblastos para obtener el desarrollo del largo radicular (23).

Una gran parte de los dientes permanentes inmaduros con fracturas coronarias y pulpa expuesta, mantienen la vitalidad pulpar porque la inflamación se limita a la superficie del tejido (24), en estos casos el tratamiento de elección suele ser la pulpotomía que consiste en la remoción de tejido pulpar a nivel coronario, buscando que el remanente radicular pulpar promueva la formación normal de la dentina en la raíz dentaria (24).

La pieza dentaria al instante de erupcionar ha conseguido un desarrollo del 60 al $80 \%$ de su raíz, y habitualmente es más susceptible a las exposiciones pulpares por lesión cariosa, debido a que los tejidos duros no se encuentran adecuadamente mineralizados, permitiendo la difusión de los ácidos más rápidamente (25); por ende, es inevitable en algunos casos llevar a cabo la terapia pulpar.

El desarrollo, la formación completa de la raíz y el cierre apical se da hasta 3 años después de la erupción dental. Los sucesos traumáticos acontecen antes de completar la formación a nivel radicular y pueden originar desde inflamación del tejido pulpar hasta necrosis. Sin embargo, es importante destacar que posterior 
a los eventos traumáticos, la formación radicular puede continuar (26).

Dentro de los materiales frecuentemente utilizados en el procedimiento de apicogénesis se encuentra, el hidróxido de calcio $\left(\mathrm{Ca}(\mathrm{OH})_{2}\right)$, el cual, fué introducido en el año 1920 por Hermann como un relleno biológico (22). Aunque se desconoce puntualmente el mecanismo por el cual provoca una barrera apical sólida, esto ocurre como consecuencia de sus propiedades antibacteriales asociadas con su alto $\mathrm{pH}(\mathrm{pH} 12)$ y la presencia de iones hidroxilo, por lo tanto, inhibe la actividad osteoclástica y previene el ingreso de tejido de inflamación o granulación en el conducto radicular $(27,28)$.

No obstante, su supuesto éxito en la terapia pulpar vital ha sido cuestionado, debido a que $\mathrm{Ca}(\mathrm{OH})_{2}$ por su $\mathrm{pH}$ alcalino produce necrosis superficial del tejido pulpar cuando es utilizó como recubrimiento pulpar directo en su estado puro (29). Estudios han mostrado que el $\mathrm{Ca}(\mathrm{OH})_{2}$ es muy tóxico para las células, por ser caustico para la pulpa, además de mostrar un efecto de disolución del tejido, lo que hace que su uso en terapias pulpares sea debatido $(30,31)$. Presenta diferentes desventajas: procedimiento versátil o variable, varias citas (hasta años), se depende del grado de colaboración por parte del paciente y existe la probabilidad de fractura debido a la formación de paredes con tejido duro delgado; a pesar de su eficacia en tratamientos de apicogénesis $(30,31)$.

Actualmente se emplea el agregado de trióxido mineral, más frecuentemente conocido como MTA, para estos procedimientos. Este material proporciona una excelente capacidad de sellado sobre la pulpa vital al no disolverse fácilmente cuando se utiliza $(32,33)$. El MTA fue introducido en 1993 por Torabinejad, en 1995 fue patentado y aprobado para procedimientos endodónticos en 1999 (32). El MTA es un polvo compuesto principalmente de silicato tricálcico, silicato dicálcico, aluminato dicálcico, sulfato dehidrato de calcio y óxido de bismuto $(34,35)$. Este material al ser hidratado se convierte en un gel coloidal que se solidifica en aproximadamente 4 horas $(36,37)$.

En diversos estudios el MTA ha reportado que produce mínima inflamación, promoviendo la odontogénesis, permitiendo que las respuestas celulares como: la migración, adhesión y multiplicación causen la regeneración del tejido pulpar (37-39). Dentro de las propiedades del material esta: capacidad de sellado, biocompatibilidad, $\mathrm{pH}$ alcalino, proliferación celular y originar un ambiente propicio para la formación de cemento, tiene efecto osteoconductivo y cementoconductivo, los fibroblastos y osteoblastos estimulan a la fosfatasa alcalina, osteocalcina e interleuquinas, induciendo a la formación del cierre apical $(38,39)$.

\section{Reporte de caso}

Paciente de 13 años y 10 meses de edad, que acude al Servicio de Odontopediatría de la Clínica dental docente de la Universidad Peruana Cayetano Heredia (UPCH), sede San Martín, por presentar dolor exacerbado a los estímulos térmicos (calor y frío) y a la masticación, En una siguiente visita el dolor se manifiesta de forma espontánea, por tal motivo es remitido al servicio de endodoncia de la UPCH para evaluación, plan de tratamiento y pronóstico del canino permanente superior izquierdo (pieza 23).

En la pieza 23 clínicamente se evidencia una lesión cariosa amplia, y tanto el paciente como su padre mencionan que la pieza dentaria presenta un defecto desde el momento de su erupción, pero no fue evaluado por un especialista, por lo cual se descarta el hecho de una fractura coronaria o caries posterior a la erupción.

Al cambio térmico da respuesta positiva moderada $(++)$, a la percusión vertical da respuesta negativa (figura 1 y figura 2). Radiográficamente se evidencia IRL c/lesión cariosa con aparente compromiso pulpar y ápice abierto, estadio de desarrollo radicular 9 de Nolla (figura 3). El diagnóstico de la pieza dentaria 23 fue pulpitis reversible sintomática y periodonto sano. Se recomendó hacer la remoción de tejido cariado convencionalmente y de producirse una exposición pulpar realizar el tratamiento de apicogénesis una vez obtenido el consentimiento de los padres, el cual tiene un pronóstico favorable.

Mientras se valoraban los exámenes radiográficos en la base de datos del paciente se observa una Rx Panorámica del año 2015 (figura 1) tomada por motivos ortodónticos en donde se evidencia que la pza 23 que estaba iniciando su proceso eruptivo presentaba un defecto de tipo PEIR (figura 2) muy pocas veces reportado en un canino permanente maxilar, este defecto ha provocado el posterior desarrollo de una lesión cariosa en dicha pieza dentaria. 
Teniendo en cuenta, que la pieza dentaria al momento de la evaluación ya se encuentra erupcionada, el tratamiento es el de remoción de caries convencional, el cual se inició con anestesia local (lidocaína al 2\% con epinefrina), aislando con dique de goma, y al realizar la eliminación de la lesión cariosa por urgencia, se generó una exposición pulpar amplia, por lo cual se decidió realizar una pulpotomía total y recubrimiento directo del remanente pulpar con $\mathrm{Ca}(\mathrm{OH})_{2}$. El acceso y la eliminación completa de la lesión cariosa se realizó con fresa redonda de diamante de alta velocidad estéril; se hizo la pulpotomía, y la asepsia de la cavidad con clorhexidina al $2 \%$; el sangrado fue controlado con una torunda de algodón estéril humedecida con solución anestésica de lidocaína al 2\% con epinefrina 1:80.000, se ejerció presión suave por 2 a 5 minutos. Luego de esto, se colocó $\mathrm{Ca}(\mathrm{OH}) 2$ (Apex$\mathrm{Cal}^{\circledR}$, Ivoclar Vivadent) por un lapso de una semana y se selló con ionómero de vidrio (figura 4).

En la segunda cita el paciente llego completamente asintomático, se procedió a colocar anestesia infiltrativa de lidocaína al 2\% con epinefrina 1:80.000, aislamiento absoluto, asepsia y antisepsia, remoción de la restauración de ionómero de vidrio, remoción de hidróxido de calcio, irrigación con clorhexidina al $2 \%$, se colocó MTA (MTA ANGELUS ${ }^{\circledR}$ ), el mismo que para su preparación se siguió las instrucciones del fabricante, y la mezcla se colocó en la entrada del conducto radicular con un espesor de 3-4 mm aproximadamente (figura 5) y se compactó con un condensador. Para el fraguado del material se colocó una bolita de algodón humedecida por un intervalo de 15 minutos y se colocó ionómero de vidrio como sellado coronal (figura 5). Se efectúo una cita de con- trol al mes, el paciente llegó asintomático, se le ejecutó la valoración clínica, evidenciando respuesta de normalidad a la palpación del fondo del vestíbulo, la percusión vertical y horizontal, finalizando la fase endodóntica correctiva (figura 6).

El paciente fue transferido al servicio de Odontología Restauradora y Estética (ORE) para la restauración definitiva, regresó a su control de 3 meses y se comprobó que el diente estaba funcional y asintomático (figura 6). El paciente regresa a los 10 meses posteriores a su tratamiento para el control clínico y radiográfico observándose cambio de coloración grisáseo a nivel cervical y formación radicular normal y cierre apical completo (figura 7).

Al cumplirse el año post tratamiento el paciente acude a la cita para su control, este presentaba clínicamente la pieza dentaria funcional y asintomática, observándose que el cambio de color era el mismo que se vio a los 10 meses a nivel cervical, a las pruebas de vitalidad pulpar y a la palpación del fondo del vestíbulo da respuestas de normalidad, radiográficamente se evidencia desarrollo, formación normal y completa a nivel radicular (figura 8).

Es de importancia considerar las respuestas a las diversas pruebas de vitalidad pulpar en dientes que han tenido este tipo de tratamientos, debido a que no siempre es posible establecer la sensibilidad ya que no existe pulpa cameral y el conducto está sellado por el MTA, lo que aísla el estímulo y no es posible fijar el grado de respuesta; para considerar en estos casos el éxito o fracaso del tratamiento tenemos que basarnos en el examen radiográfico y la sintomatología que pueda reportar o no el paciente.

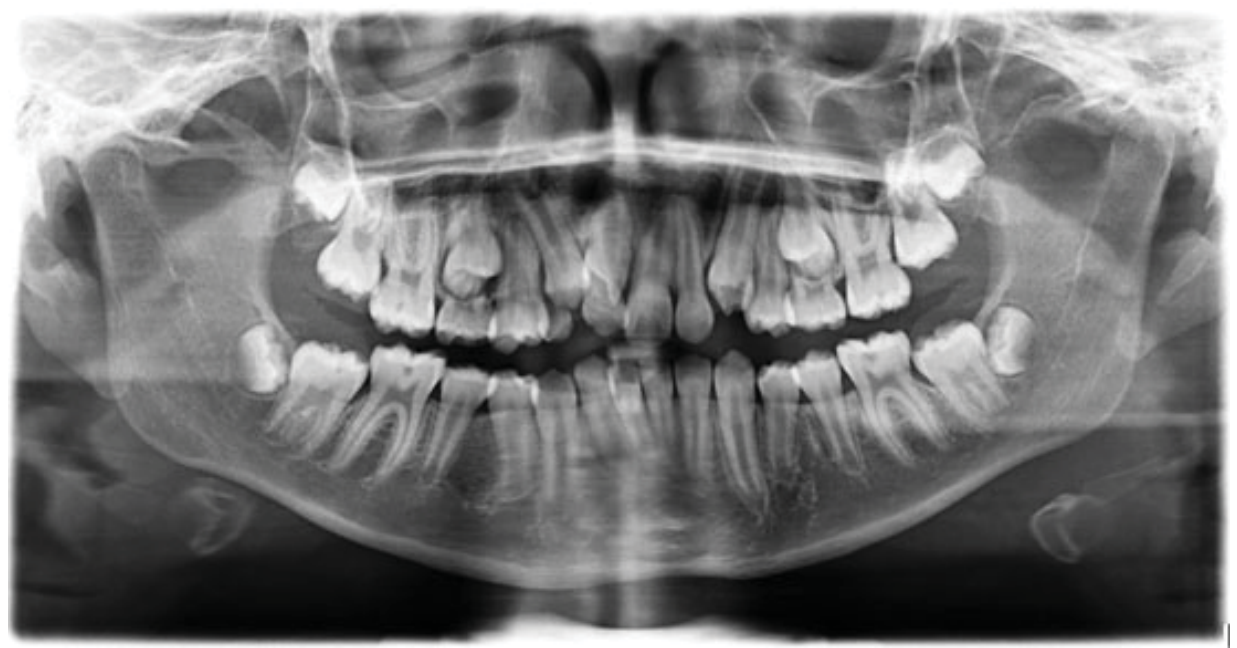

Figura 1. Rx Panorámica año 2015 


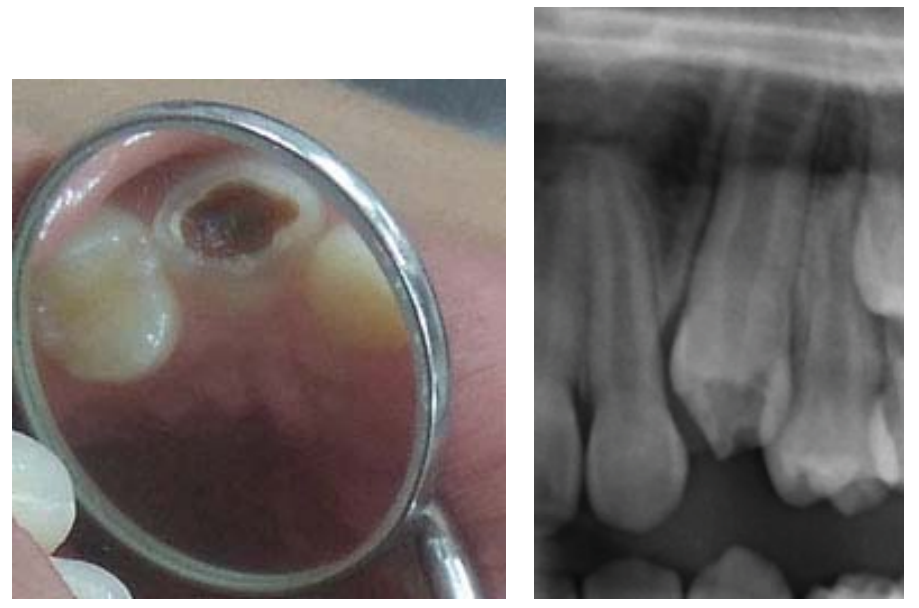

Figura 2. Destrucción Coronal-lesión cariosa PEIR (pza 23)

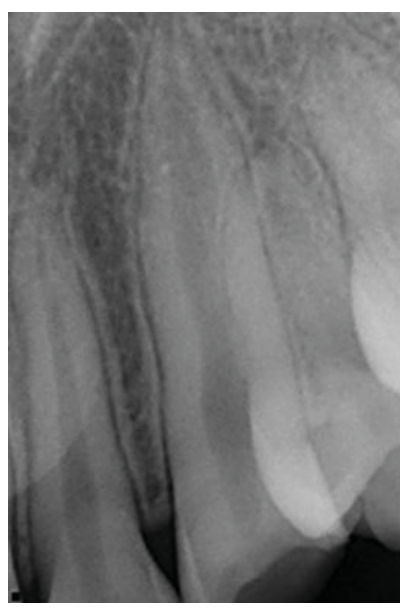

Figura 3. Rx Inicial de Diagnóstico

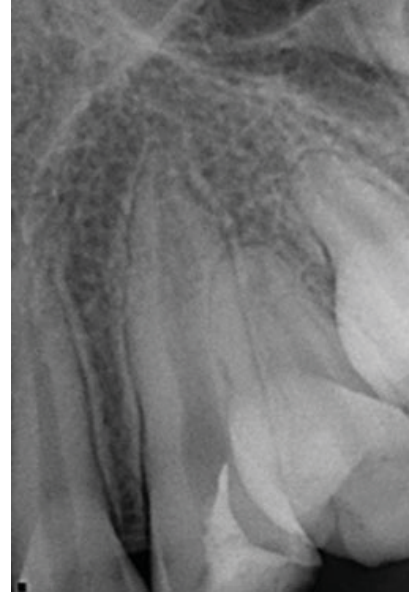

Figura 4. Rx sellado $\mathrm{Ca}(\mathrm{OH}) 2$
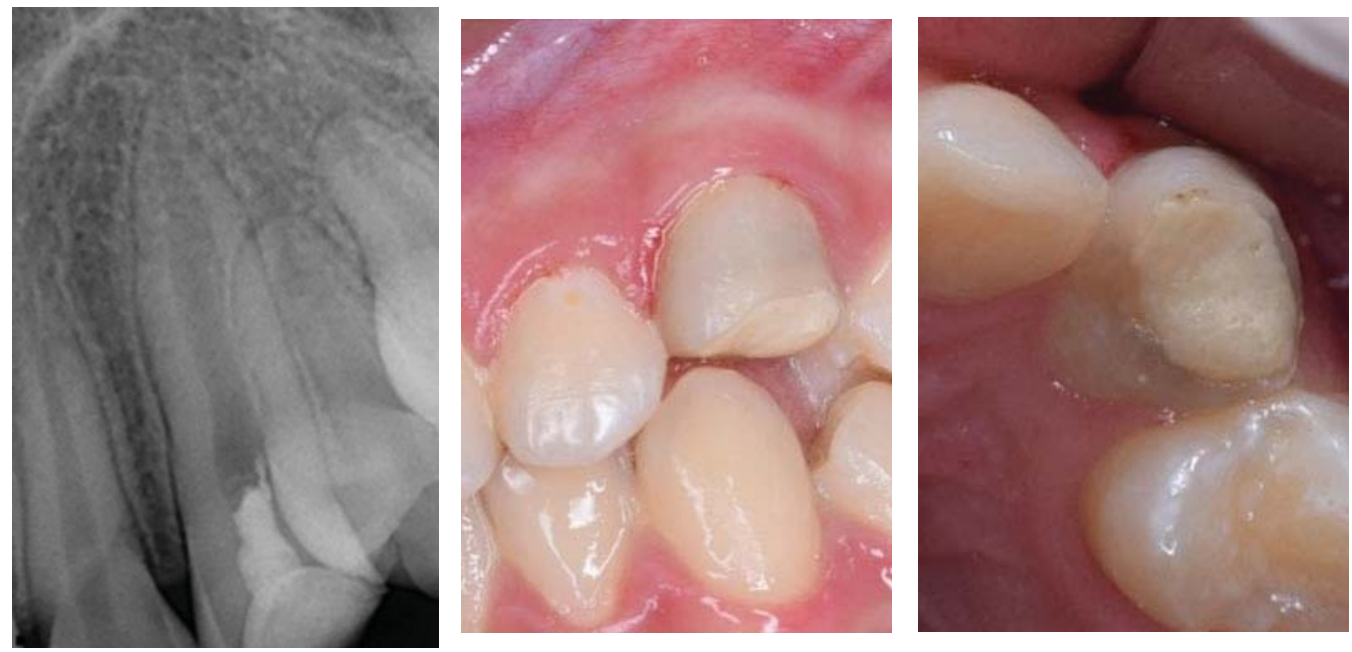

Figura 5. Rx sellado MTA e Imagen Restauración con Ionómero 

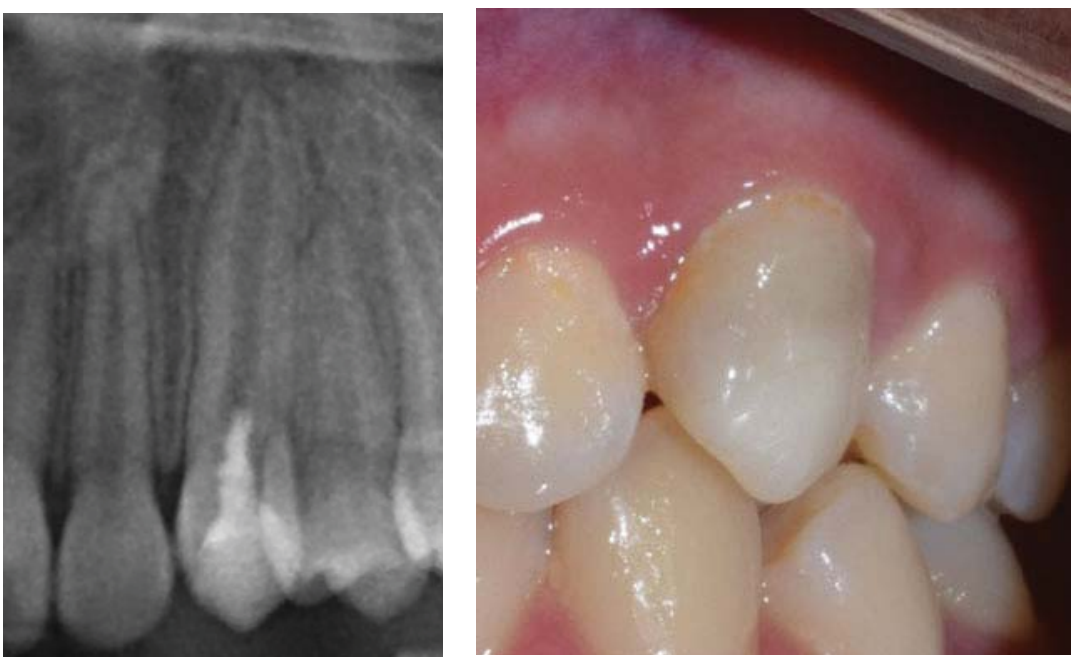

Figura 6. A. Rx Control a los 3 meses. B. Imagen Restauración Definitiva Resina
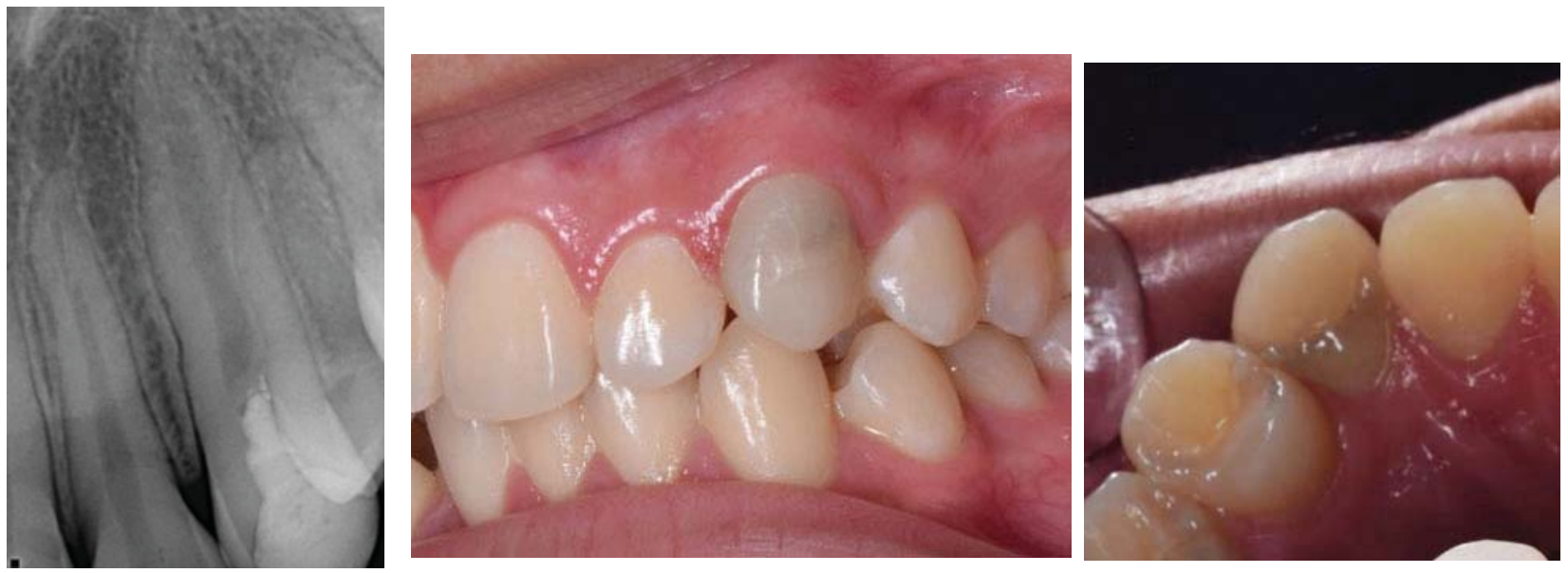

Figura 7. Rx Control y Clínico a los 10 meses. Cambio de Coloración a Nivel Cervical
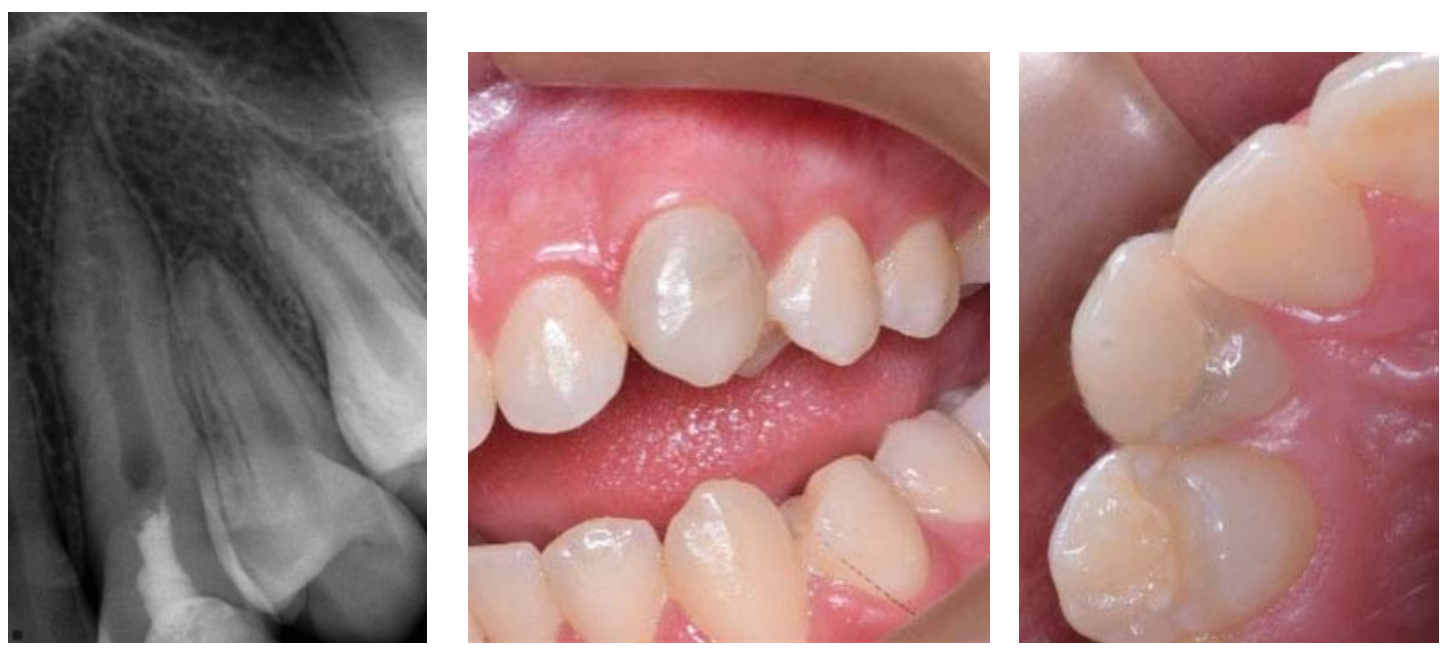

Figura 8. Control Clínico y Radiográfico Anual. Cambio de Coloración a Nivel Cervical.
A. Cara Vestibular
B. Cara Palatina 


\section{DISCUSIÓN}

Es importante realizar un diagnóstico oportuno frente a una resorción intracoronal pre eruptiva, para planificar y realizar un tratamiento inmediato cuando se requiera prevenir la afectación pulpar, incluso el abordaje puede ser quirúrgico restaurador si se detecta en una etapa intraósea, aunque suele ser muy poco frecuente ya que su hallazgo suele ser incidental.

La conservación de la pulpa es considerablemente importante por diferentes razones; y éstas son: continuar el crecimiento y desarrollo en aquellas piezas que no han completado la formación de la porción radicular, permitir a los odontoblastos crear un puente de dentina entre la pulpa y el apósito del material y mantener la vitalidad pulpar.

El presente reporte de caso se centró en el uso de materiales dentales para salvaguardar la vitalidad de la pulpa en una pieza dentaria joven, con controles de seguimiento durante 1 año, observándose el comportamiento clínico y radiográfico de la pieza dental que se trató con MTA.

Según Simon et al, los casos tratados con procedimientos de apexificación han reportado una tasa de éxito hasta el $100 \%$; indicando que el lapso de tiempo promedio es de 12 a 19 meses necesario para la formación de una barrera apical (40).

Se usó hidróxido de calcio porque ha sido el material estándar para los tratamientos de preservación de la pulpa (recubrimiento pulpar indirecto, recubrimiento directo de pulpa y pulpotomía) durante muchos años (41).

Actualmente existe suficiente soporte literario en el uso de MTA para realizar los procedimientos de apicogénesis, ya que cuenta con excelentes propiedades como su alto grado de biocompatibilidad, buen sellado y una buena adaptación marginal entre otras.

Según Alqader et al., en su revisión de artículos concluyeron que las diferencias en los materiales de recubrimiento pulpar y de restauración, no afectaron significativamente las tasas de éxito (42).

Hay evidencia de que la pulpectomía no es el único tratamiento posible para los dientes permanentes vitales con exposición a la pulpa debido a caries dental. En el estudio realizado por Linsuwanont et al.,se con- cluye que la pulpotomía con MTA es una opción para dientes permanentes vitales con exposición pulpar por caries, incluidos los dientes con signos y síntomas irreversibles; mostrando resultados exitosos con una tasa de éxito del 87,3\% (43).

\section{CONCLUSIONES}

Este caso demuestra que los defectos causados por PEIR pueden afectar el tejido pulpar en un corto período de tiempo, además la detección temprana brinda un pronóstico favorable con el tratamiento adecuado. Recalcando que es una patología de escasa frecuencia especialmente a nivel de caninos permanentes jóvenes.

Existe literatura limitada disponible con respecto a su uso en dientes anteriores permanentes jóvenes. Sin embargo, el éxito de la terapia de pulpa vital con MTA depende de la selección adecuada de casos y la técnica de manejo del diente más que del material en sí. El MTA como agente de pulpotomía ha mostrado resultados favorables en casos de molares temporales y permanentes y en el caso clínico mostrado en un canino permanente joven.

\section{Correspondencia}

Adriana Bravo Zhunio

Correo electrónico: adriana.bravo.z@upch.pe

\section{REFERENCIAS BIBLIOGRÁFICAS}

1. Brooks JK. An unusual case of idiopathic internal root resorption beginning in an unerupted permanent tooth. J Endod. 1986; 12(7):309-10.

2. Seow WK. Pre-eruptive intracoronal resorption as an entity of occult caries. Pediatr Dent 2000; 22:370-6.

3. Seow WK. Multiple pre-eruptive intracoronal radiolucent lesions in the permanent dentition: case report. Pediatr Dent 1998; 21: 195-198.

4. Manmontri C, Mahasantipiya PM, Chompu-Inwai P. Preeruptive intracoronal radiolucencies: Detection and nine years monitoring with a series of dental radiographs. Case Rep Dent. 2017;2017:6261407.

5. Hemani K, Deepa G. Pre eruptive intracoronal resorption- a review. International Journal of Current Advanced Research.2017; 6: 3589-3591.

6. Sawle RF, Andlaw RJ. Has occlusal caries become more difficult to diagnose? A study comparing clinically undetected lesions in molar teeth of 14-16 year 
old in 1974 and 1982. Br Dent J. 1988;164(7):209-211.

7. Creanor SL, Russell JI, Strang DM, Stephen KW, Burchell CK. The prevalence of clinically undetected occlusal dentine caries in Scottish adolescents. $\mathrm{Br}$ Dent J. 1990; 169(5):126-129

8. Kidd EA, Naylor MN, Wilson RF. The prevalence of clinically undetected and untreated molar occlusal dentine caries in adolescents in the Isle ofWight. Caries Res. 1992; 26(5):397-401

9. Weerheijm KL, Groen HJ, Bast AJ, Kieft JA, Eijkman MA, van Amerongen WE. Clinically undetected occlusal dentine caries: A radiographic comparison. Caries Res. 1992; 26(4):305-309.

10. Manan NM, Mallineni SK, King NM. Case report: idiopathic pre-eruptive coronal resorption of a maxillary permanent canine. Eur Arch Paediatr Dent. 2012; 13(2):98-101.

11. Stafne EC, Austin LE. Resorption of embedded teeth. J Am Dent Assoc. 1945; 32: 1003-1009.

12. Seow WK, Lu PC, McAllan LH. Prevalence of pre-eruptive intracoronal dentin defects from panoramic radiographs. Pediatr Dent.1999;21(6):332-9.

13. Seow WK, Wan A, McAllan LH. The prevalence of pre-eruptive dentin radiolucencies in the permanent dentition. Pediatr Dent. 1999;21(1):26-33.

14. Özden B, Acikgoz A. Prevalence and characteristics of intracoronal resorption in unerupted teeth in the permanent dentition: a retrospective study. Oral Radiol. 2009;25(1):6-13.

15. Al-Tuwirqi A, Seow WK. A controlled study of pre-eruptive intracoronal resorption and dental development. J Clin Pediatr Dent. 2017;41(5):374-380.

16. Ari T. Management of "hidden caries": a case of severe pre-eruptive intracoronal resorption. J Can Dent Assoc. 2014; 80:e59.

17. Konde S, Sri Darshini CS, Agarwal M, Peethambar P. Unrevealed caries in unerupted teeth: a prevalence study. Contemp Clin Dent. 2018;9(Suppl 2):S305-S308.

18. McNamara CM, Foley T, O'Sullivan VR, Crowley N, McConnel RJ. External resorption presenting as an intracoronal radiolucent lesion in a pre-eruptive tooth. Oral Dis. 1997; 3(3:)199-201

19. Spierer WA, Fuks AB. Pre-eruptive intra-coronal resorption: controversies and treatment options. J Clin Pediatr Dent. 2014;38(4):326-8.

20. Rutar JE. Paediatric dentistry: coronal radiolucency. Case reports. Aust Dent J. 1997;42(4):221-4

21. Seow WK. Diagnosis and management of unusual dental abscesses in children. Aust Dent J. 2003;48(3):156-68.

22. Hermann BW. Biologische Wurzelbehandlung: Frankfurt arn Main. Deutsche Zahnarztliches Wochenschrift.1936; 38: 461-5

23. ChapleAM, Herrero L. Generalidades del agregado de trióxido mineral (mta) y su aplicación en odontología. Revisión de Literatura. Acta Odontol Venez. 2007; 45(3):0-0.

24. Odontología Virtual. ENDODONCIA: Apexificación y Apicogénesis. Definición de términos. Lima: Odontología Virtual; 2016. (Fecha de acceso 15 de marzo del 2019) Disponible en: https://www.odontologiavirtual.com/2011/10/apexificacion-y-apexogenesis-definicion.html

25. Villena H. Materiales utilizados en la terapia endodóntica de dientes primarios. En: Villena H. Endodoncia pediátrica. Lima: Universidad Peruana Cayetano Heredia; 2005. p. 206.

26. Rafter M. Apexification: a review. Dent Traumatol. 2005;21(1):1-8.

27. Tait CME, Ricketts DNJ, Higgins AJ. Weakened anterior roots--intraradicular rehabilitation. Br Dent J. 2005;198(10):609-617.

28. Cehreli ZC, Sara S, Uysal S, Turgut MD. MTA apical plugs in the treatment of traumatized immature teeth with large periapical lesions. Dent Traumatol. 2011;27(1):59-62. 12

29. McDonald RE, Avery DR, Dean JA. Treatment of deep caries, vital pulp exposure and pulpless teeth. Dentistry for the child and adolescent. 8a. ed. St. Louis: Mosby; 2004. p. 389-412.

30. Anusavice KJ. Biocompatibility of dental materials. Phillip's science of dental materials. 10th. ed. Philadelphia: WB Saunders; 1996. p. 57-110.

31. Holland R, de Souza V, Nery MJ, Otoboni Filho JA, Bernabe PF, Dezan JE. Reaction of rat connective tissue to implanted dentin tubes filled with mineral trioxide aggregate or calcium hydroxide. J Endod. 1999; 25: $161-6$

32. Torabinejad M, Chivian N. Clinical applications of mineral trioxide aggregate. J Endod. 1999; 25: 197 205.

33. Torabinejad M, Hong CU, McDonald F, Pitt-Ford TR. Physical and chemical properties of a new root-end filling material. J Endod. 1995; 21: 349-53.

34. Maroto M, Barbería E, Vera V, García-Godoy F. Mineral trioxide aggregate as pulp dressing agent in pulpotomy treatment of primary molars: 42-month clinical study. Amer J Dent. 2007; 20(5): 283-6.

35. Hakki SS, Bozkurt SB, Hakki EE, Belli S. Effects of mineral trioxide aggregate on cell survival, gene expresión associated with mineralized tissues, and biomineralization of cementoblasts. J Endod. 2009; 35: 513-9.

36. Tait CME, Ricketts DNJ, Higgins AJ. Weakened anterior roots--intraradicular rehabilitation. Br Dent J. 2005;198(10):609-617.

37. Cehreli ZC, Sara S, Uysal S, Turgut MD. MTA apical plugs in the treatment of traumatized immature teeth with large periapical lesions. Dent Traumatol. 
2011;27(1):59-62.

38. Guven EP, Yalvac ME, Sahin F, Yazici MM, Rizvanov AA, Bayirli G. Effect of dental materials calcium hydroxide-containing cement, mineral trioxide aggregate, and enamel matrix derivative on proliferation and differentiation of human tooth germ stem cells. J Endod. 2011;37(5):650-656.

39. Felippe WT, Felippe MCS, Rocha MJC. The effect of mineral trioxide aggregate on the apexification and periapical healing of teeth with incomplete root formation. Int Endod J. 2006;39(1):2-9

40. Simon S, Rilliard F, Berdal A, Machtou P. The use of mineral trioxide aggregate in one-visit apexification treatment: a prospective study. Int Endod J. 2007;40(3):186-197.

41. Brizuela C, Ormeño A, Cabrera C, et al. Direct pulp capping with calcium hydroxide, mineral trioxide aggregate, and biodentine in permanent young teeth with caries: A randomized clinical trial. J Endod. 2017;43(11):1776-1780. doi: 10.1016/j. joen.2017.06.031

42. Alqaderi H, Lee CT, Borzangy S, Pagonis TC. Coronal pulpotomy for cariously exposed permanent posterior teeth with closed apices: A systematic review and meta-analysis. J Dent. 2016; 44:1-7.

43. Linsuwanont $\mathrm{P}$, Wimonsutthikul K, Pothimoke U, Santiwong B. Treatment outcomes of mineral trioxide aggregate pulpotomy in vital permanent teeth with carious pulp exposure: The retrospective study. J Endod. 2017;43(2):225-230.

Recibido: 13-08-2018

Aceptado: 16-12-2018 